\title{
Information Compressed and Transmitted and Reconstructed System of CGH with LOCO-I Image Processing and Fraunhofer Transforming Technique
}

\author{
Non-member Guanglin Yang (Osaka City University) \\ Member Eiji Shimizu (Osaka City University)
}

\begin{abstract}
In this paper, the information compressed and transmitted and reconstructed software system (ICTRS) of Computer generated hologram (CGH) has been designed and established which can be widely applied by computer operators. This system structure can be applied to resolve the remote signal processing using the digital filter of $\mathrm{CGH}$. It is a new system structure, and the size of image displayed is bigger (i.e., $7.11 \times 7.11\left(\right.$ inch $\left.\left.^{2}\right)\right)$. In this system, LOCO-I image encoding technique and the image reconstruction technique using Fraunhofer transforming algorithm have been adapted. This processing method is a "lossless" or "near-lossless" compression algorithm whose compression ratio can be achieved to $1: 5$ (i.e., the compression ratio is $18.7806 \%$ ), and the image of processed CGH can be effectively reconstructed by a computer. In experiments, we have mainly discussed and analyzed the amplitude and phase variation of CGH's pixels. In detail, re have explained why the image quality of processed CGH can be influenced by the pixel's amplitude and phase varied. The image quality of processed CGH has been compared with the image quality of original $\mathrm{CGH}$, and the reconstructed image quality of processed CGH has been compared with the reconstructed image quality of original CGH in ICTRS. Finally, Compression ratio (R), Mean squared error (MSE) and Pear signal to noise ratio (PSNR) have been precisely calculated and analyzed to evaluate the reconstructed images quality of processed CGH and the image quality of processed CGH. The better compression and transmission and reconstruction algorithm model of CGH can be determined by the distortion measure. In this system, the image quality of processed CGH has been relatively improred. This method of processing $\mathrm{CGH}$ has been effectively rerified by experiments
\end{abstract}

Keywords: ICTRS. CGH. LOCO-I. Fraunhofer transforming technique. compressed and transmitted and reconstructed.

\section{Introduction}

In this paper, the information compressed and transmitted and reconstructed software system of CGH has been designed and established as shown in Fig. 1. It can be applied in the remote signal processing using the digital filter of CGH. In this system. LOCO-I (Low Complexity Lossless Compression for Images) image processing algorithm model $12 \sim(4)$ and Fraunhofer transforming technique isinia, have been adapted to process CGH. This method of processing CGH is different from JPEG baseline image processing and Fresnel transforming technique 11 . It is the "lossless" or "nearlossless" compression and transmission technique. The diffraction-pattern calculation can be further simplified and the restrictions can be more stringent than those used in the Fresnel approximations are adapted. Using this system, a few problems have been discussed when CGH are compressed and transmitted and reconstructed

In researching, some documents about CGH processed have been investigated. Their processing methods are about the electronic hologram hardware display system illiwi13;. However, at present the computer has quickly been dereloped and widely been applied in the every field, and CGH has the potential application Thus we think whether we should use the computer software to complete the total process of CGH compressed and transmitted and reconstructed.

The aim of processing $\mathrm{CGH}$ is that the information content of CGH is compressed and quickly transmitted in electronic system (e.g., the Internet). Therefore, if the algorithm model exists the error problems to process CGH, the image of CGH could not be effectively reconstructed after compression and transmission. So it is rery important that a suitable compression algorithm model is chosen for CGH. Moreover the image processing theories have told us, the image compressed is to remore the redundancy information of image. The redundancy of processed $\mathrm{CGH}$ is the interpixel redundancy and the coding redundancy. Thus we must choice a suitable encoding algorithm to resolve these problems.

It is well known that CGH is a collection of special optical element. During the processing, if the lost information of $\mathrm{CGH}$ exceeds certain limit value, its original information distribution (i.e.. the amplitude and phase functions) will be varied. In terms of the Detour phase holograms principle of B. R. Brown and $A$. 


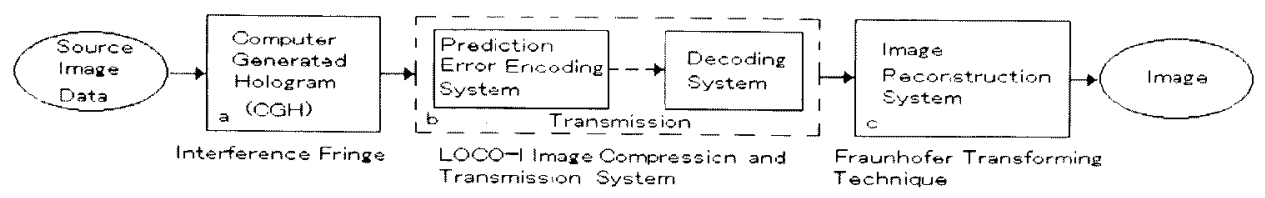

Fig. 1. Information compressed and transmitted and reconstructed system structure of CGH using LOCO-I image processing and Fraunhofer transforming technique.

IV. Lohmann, we can understand, the size variation of CGH pixels is determined by amplitude function, the position variation of CGH pixels is determined by phase function. Thus in compression and transmission, if the pixel's size can be reduced (i.e.. the limit value of $\mathrm{CGH}$ compressed can t be exceeded), and the pixel's position can be restored after CGH compressed, the image of CGH can be effectively compressed and transmitted and reconstructed.

On the other hand, in ${ }^{11}$, our experiment results have proved that the phase information of CGH is more important than the amplitude information of $\mathrm{CGH}$. The amplitude information variation of $\mathrm{CGH}$ pixels only influences the image intensity, but the original information distribution of $\mathrm{CGH}$ isn't distorted. However, if the phase information of $\mathrm{CGH}$ pixels is raried, it will disturb the information distribution of CGH. Thus, we must choice an encoding algorithm that it will only compress the amplitude information of CGH pixels, but the phase information of $\mathrm{CGH}$ pixels will be lightly reduced (or it is said that the image of the processed CGH can be reconstructed by Fraunhofer transforming algorithm). Through experiments, we have found that LOCO-I image encoding algorithm can satisfy these requirements. To adapt LOCO-I algorithm model, the compression ratio of $\mathrm{CGH}$ can be improved. and it can achieve the near-lossless amplitude and phase information to compress the redundancy information contained in the original $\mathrm{CGH}$. And the information of processed $\mathrm{CGH}$ is less influenced in compression and transmission and reconstruction.

The system structure of ICTRS and the method of processing $\mathrm{CGH}$ are presented in this paper. The algorithm principle of $\mathrm{CGH}$ is described in Sect.2. LOCO-I encoding algorithm model is described in Sect.3. The image-reconstructed algorithm of $\mathrm{CGH}$ is described in Sect.t. The experiment analyses, discussion and the conclusion are respectively described in Sect 5 and 6 .

\section{Computer Generated Hologram}

According to the theory of E. N. Leith and J. Upatnieks 1641 in off-axis reference beam holograms. we have generated an on-axis reference beam hologram of 9 points with a computer. That is, the amplitude and phase transmittance of a hologram recorded under ideal conditions is proportional to

$$
\begin{aligned}
I(x, y)= & {[r(x, y)+a(x, y)][r(x, y)+a(x, y)]^{*} } \\
= & \mid R(x, y) \exp [j o(x, y)] \\
& +\left.f(x, y) \exp [j \iota \cdot(x, y)]\right|^{2}
\end{aligned}
$$

Table 1. Experiment data of CGH about 9 object points.

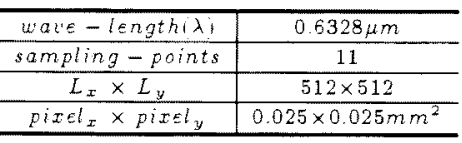

$$
\begin{aligned}
& =R(x, y)^{2}+A(x, y)^{2} \\
& +2 R(x, y) A(x, y) \cos [o(x, y) \\
& -\zeta(x, y)] . \ldots \ldots \ldots \ldots \ldots \ldots
\end{aligned}
$$

In Eq.(1), $r(x, y)=R(x, y) \exp [j o(x, y)]$ represents the tilted reference wave function and $a(x, y)=$ $A(x, y) \exp [j z(x, y)]$ is the object wave function. $I(x, y)$ is the resulting intensity variation of the interference pattern between the two waves. In experiment we have chosen that the positions of the reference beam $r(x, y, z)$ and the object wave $a(x, y, z)$ respectively are $r(0,0,1500(\mathrm{~mm}))$ and $a(0,0,150(\mathrm{~mm}))$.

In terms of Eq.(1). we have made a CGH of 9 points and its histograms with a computer, and Fraunhofer transform technique has been adapted to reconstruct the original object (i.e., 9 points) as shown in Table 1 and Fig. $\bar{T}, 10$ and 12 .

\section{LOCO-I Image Processing Model}

LOCO-I is a lossless compression algorithm for continuous-tone images that combine the simplicity of Huffman coding with the compression potential of context models. The algorithm uses a non-linear predictor with rudimentary edge detecting capability, and is based on a simple fixed context model, determined by quantized gradients. A small number of free statistical parameters can be used to approach the capability of the more complex universal context modeling techniques for capturing high-order dependencies, without context dilution. The model is tuned for efficient performance in conjunction with a collection of Huffman codes. which is realized with an adaptive, symbol-wise, Golomb-Rice code. In one pass, LOCO-I attains compression ratios similar or superior to those obtained with state-of-the-art schemes based on arithmetic coding and without recourse to the higher complexity arithmetic coders

LOCO-I follows a more traditional predictor-modelercoder structure, in the use of an extremely simple explicit formula for Golomb-Rice parameter estimation and in the use of an embedded alphabet extension to reduce code redundancr. And the prediction modeling units are based on the causal template depicted, where $x$ denotes the current pixel and a. b.c and d are neigh- 


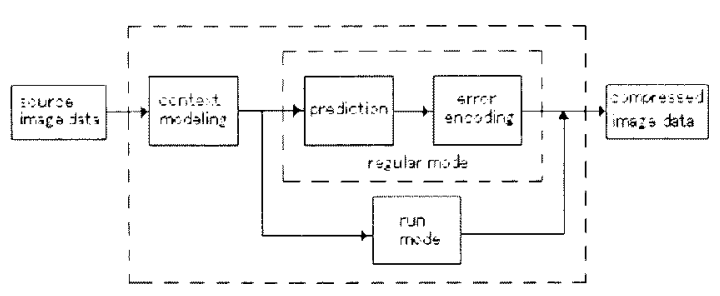

Fig. 2. Lossless encoder simplified diagram

boring pixels in the relative positions as shown in Fig. 3 The dependence of $a, b, c$ and $d$ on the time index $i$ has been deleted from the notation for simplicity

In a context model scheme, reducing the number of parameters is a kev objective. In a sequential formulation, the goal is to avoid the context dilution. while in a two-pass scheme LOCO-I can be expected to reduce unnecessary table overhead. The total number of parameters in the model depends on the number of free parameters defining the coding distribution at each context and on the number of contexts. In fact. LOCOI has been considered by the ISO/ITU committee as a replacement for the current lossless standard in lowcomplexity applications (2i (5)

3.1 Coding Principles of LOCO-I Algorithm Model In LOCO-I, a source image is input to the encoder sample after sample in a pre-defined scan pattern, and lossless image compression is formulated as an inductive inference problem as follows. When coding the current sample, after having scanned past data. inferences can be made on the value of this sample by assigning a conditional probability $\rho$ for the value of the current image sample, conditioned on previously received samples. This inference method is called modeling. The minimum average code length contribution of the current sample is $-\log _{2}(\rho)$. For near-lossless image compression this principle is modified to use reconstructed values of the preceding samples (instead of the original values) as conditioning data. During the encoding process, shorter codes are assigned to more probable events. The decoder can reconstruct the conditional probability used to encode the current samples, since it depends only on already decoded data 2 i is

\subsection{Encoding Process Steps}

3.2.1 Context Modeling The encoding process steps of LOCO-I are described in Fig.2. In this international standard, the modeling approach used is based on the notion of "context". In context modeling, each sample value is conditioned on a small number of neighboring samples. The context modeling procedure determines a probability distribution used to encode the current sample, whose position. $\mathrm{x}$, is shown in Fig.3. The context is determined from four neighborhood reconstructed samples at positions a. b. c, and d of the same component, as shown in Fig.3. From the ralues of the reconstructed samples at a, b, c, and d, the context first determines if the information in the sample $x$ should be encoded in the regular or run mode 5 ;

(1) The regular mode is selected when the context estimates samples are not rery likely to be identical (for

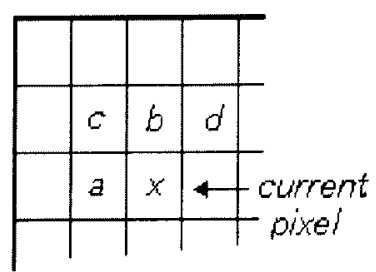

Fig. 3. Causal template used for context modeling and prediction.

lossless coding) or nearly identical within the tolerances required (for near-lossless coding)

(2) The run mode is selected when the context estimates that successive samples are very likely to be either identical (for lossless coding) or nearly identical within the tolerances required (for near-lossless coding)

3.2.2 Regular Mode(include: Prediction and Error Encoding) In the regular mode, the context determination procedure is followed by a prediction procedure. The predictor combines the reconstructed ralues of the three neighborhood samples at positions $a, b$. and $c$ to form a prediction of the sample at position $\mathrm{x}$ as shown in Fig. 3. The prediction error is computed as the difference between the actual sample value at position $\mathrm{x}$ and its predicted value. This prediction error is then corrected by a context-dependent term to compensate for systematic biases in prediction. In the case of nearlossless coding, the prediction error is then quantized.

The corrected prediction error (further quantized for near-lossless coding) is then encoded using a procedure derived from Golomb coding. The Golomb coding procedures specified in this International Standard depend on the context determined by the values of the samples at positions $a, b, c$, and $d$ as well as prediction errors previously encoded for the same context $44(5)$

3.2.3 Run Mode If the reconstructed values of the samples at $a, b, c$ and $d$ are identical for lossless coding, or the differences between them (the gradients. specified in (5i) are within the bounds set for nearlossless coding, the context modeling procedure selects the run mode and the encoding process skips the prediction and error encoding procedures. In run mode, the encoder looks, starting at $\mathrm{x}$. for a sequence of consecutive samples with ralues identical (or within the bound specified for near-lossless coding) to the reconstructed ralue of the sample at a. This run is ended by a sample of a different value for one which exceeds the bound specified for near-lossless coding), or by the end of the current line, whichever comes first. The length information, which also specifies one of the above two run-ending alternatives, is encoded using a procedure specified in 15i, which is extended from Golomb coding but has improved performance and adaptability.

3.3 Decoding Process Steps The encoding - and decoding processes are approximately symmetrical. It is also said that the decoding processes are the encoding inverse processes. Therefore, the decoding process is not repeatedly described in this section. The detail of the decoding process is in 445 i 


\section{Image Reconstructed of CGH with Fraun- hofer Transforming Technique}

According to above theories in Sect.1, we can understand the algorithm of CGH compressed and transmitted and reconstructed must satisfy two conditions. That is, both the variation of amplitude and the variation of phase can be controlled in a limit value respectively as CGH compressed. Thus there are two limit values when the pixels of $\mathrm{CGH}$ are reduced. The limit value of amplitude compressed can be easily found with experiments (i.e. to see Table 2 and Fig. $12 \sim 14$ ). However. the limit value of phase reduced can't be easily found out through experiments. Because there isn t a suitable method that can precisely determine the position variation of pixel. And the phase variation of compressed pixels is relatively less than the amplitude variation of compressed pixels. In addition, this processing method is a randomization. So it is more difficult to determine the position variation of CGH pixels.

Fortunately, we have found out a method that can resolve this problem. That is. Fraunhofer transforming technique is adapted to reconstruct the images of the original $\mathrm{CGH}$ and the processed $\mathrm{CGH}$, and then to compare the positions of their information distribution with each other. Because Fraunhofer transforming technique is a plane wave reconstruction algorithm, applying it to calculate, the reconstructed image of $\mathrm{CGH}$ is a black/white information distribution. Thus the positions of information distribution can be easily recognized by human risual system (i.e., to see Fig.10,11) That is one of the reasons why Fraunhofer transforming technique is adapted in this system

Therefore, in transforming algorithm, if the diffractionpattern calculation can be further simplified and the restrictions can be more stringent than those used in the Fresnel approximations are adopted, in particular. it was seen earlier that in the region of Fresnel diffraction the observed field strength $h\left(x_{2}, y_{2}\right)$ can be found from a Fourier transform of the product of the aperture distribution $h\left(x_{1}, y_{1}\right)$ with a quadratic phase function $\exp \left[j(k / 2 z)\left(x_{1}^{2}+y_{1}^{2}\right)\right]$. If in addition the stronger assumption is adopted.

$$
z \gg \frac{k}{2}\left(x_{1}^{2}+y_{1}^{2}\right)_{\max }
$$

then the quadratic phase factor is approximately unity over the entire aperture, and the observed field distribution can be found directly from a Fourier transform of the aperture distribution itself. Thus in the region of Fraunhofer diffraction

$$
\begin{aligned}
h\left(x_{2}, y_{2}\right)= & \frac{\exp (j k z)}{j \lambda z} \exp \left[\frac{j k}{2 z}\left(x_{2}^{2}+y_{2}^{2}\right)\right] \\
& \cdot \iint_{-x}^{+x} h\left(x_{1}, y_{1}\right) \\
& \cdot \exp \left[-\frac{j k}{z}\left(x_{2}, x_{1}+y_{2} y_{1}\right)\right] d x_{1} d y_{1}
\end{aligned}
$$

Aside from the multiplicative factors preceding the integral, this expression is simply the Fourier transform

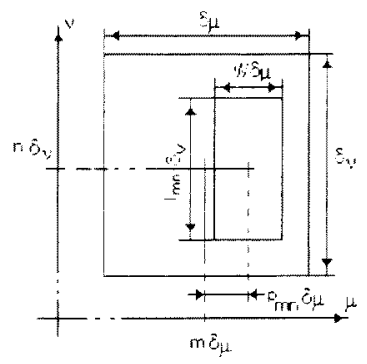

Fig. 4. Sampling unit.

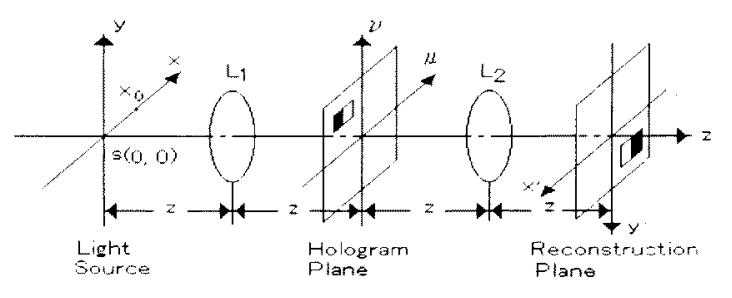

Fig. 5. Optical image reconstruction construction of $\mathrm{CGH}$.

of the aperture distribution, evaluated at frequencies $\left(\mu=x_{2} / \lambda z, \nu=y_{2} / \lambda z\right)$. That is

$$
\begin{aligned}
h\left(x_{2}, y_{2}\right) & =\left.A \cdot F\left\{h\left(x_{1}, y_{1}\right)\right\}\right|_{\substack{\mu=x_{2} / \lambda x \\
\nu=y_{2} / \lambda x}} \\
& =A \cdot H(\mu, \nu) \ldots \ldots \ldots \ldots
\end{aligned}
$$

where $A$ is defined as $\frac{\exp (j k z i}{j \lambda z} \exp \left[\frac{j k}{2 z}\left(x_{2}^{2}+y_{2}^{2}\right)\right]$. Therefore, if $H(\mu, \nu)$ can be inverse Fourier transformed. the object wave of $h\left(x_{2}, y_{2}\right)$ can be reconstructed by a computer.

In holography, the object's diffraction wave $h\left(x_{2}, y_{2}\right)$ which is recorded on the hologram plane is Fraunhofer transform function of the object's amplitude distribution $h\left(x_{1}, y_{1}\right)$ in the distance $z$. Therefore, it can be expressed by Eq. (4), where $k$ is the ware number $2 \pi / \lambda$. If the plane reference wave would be adapted to illuminate the hologram plane in Fig.5, then the original image of object can be reconstructed in the distance $z+8199$. Therefore, according to holographic theory ifu', every sampling unit function of hologram plane can be written by

$$
\operatorname{rect}\left[\frac{\left.\mu-p_{m n} \hat{\delta}_{\mu}\right)}{\Pi^{r} \delta_{\mu}}\right] \operatorname{rect}\left[\frac{\nu}{l_{m n} \delta_{\nu}}\right]
$$

the function rect $(x)$ is given by

$$
\operatorname{rect}(x)= \begin{cases}1 & \text { for }|x| \leq \frac{1}{2} \\ 0 & \text { otherwise. }\end{cases}
$$

The equation of hologram plane function can be directly. written by

$$
\begin{aligned}
H(\mu, \nu)= & \sum_{m} \sum_{n} \operatorname{rect}\left[\frac{\mu-\left(m+p_{m n}\right) \delta_{\mu}}{\| \delta_{\mu}}\right] \\
& \cdot \operatorname{rect}\left[\frac{\nu-n \delta_{\nu}}{l_{m n} \delta_{\nu}}\right] \ldots \ldots \ldots \ldots
\end{aligned}
$$




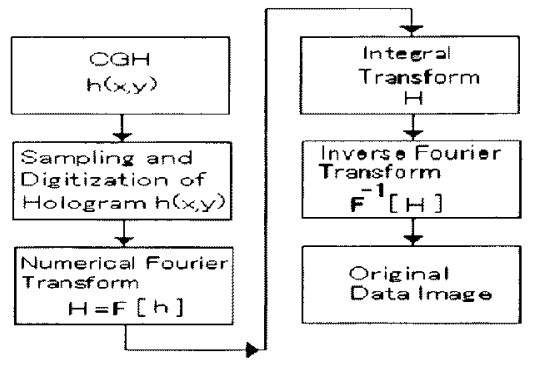

Fig. 6. Fraunhofer transform algorithm flow diagram.

As shown in Fig.4, 5, the rectangular aperture is drawn inside each cell in hologram plane, each aperture is determined by three parameters: its height $l_{m n}$, its width $\mathrm{II}$, and its center with respect to the center of the cell $p_{m n}, \delta_{\mu}$ and $\delta_{\nu}$ are the sampling distances along the $\mu$ and $\nu$ coordinates. The index $m, n$ indicates the relative location of the cell in the hologram plane.

If CGH is placed in the optical system of Fig.5, then the hologram is illuminated with a plane wave $\exp \left[j 2 \pi x_{0} \mu\right]$ in $x_{0}$. Lens $L_{2}$ performs the inverse Fourier transformation to produce the wavefront $f(x, y) \exp [j o(x, y)]$ at the back focal plane of lens $L_{2}$. The wavefront $h(x, y)$ at the back focal plane of lens $L_{2}$ is

$$
\begin{aligned}
h(x, y)= & \iint_{-\infty}^{+\infty} H(\mu, \nu) \\
& \cdot \exp \left[j 2 \pi x_{0} \mu\right] \exp [j 2 \pi(\mu x+\nu y)] d \mu d \nu \\
= & \sum_{m} \sum_{n} l_{m n} W \delta_{\mu} \delta_{\nu} \operatorname{sinc}\left[l_{m n} y \delta_{\nu}\right] \\
& \cdot \operatorname{sinc}\left[W\left(x+x_{0}\right) \delta_{\mu}\right] \exp \left[j 2 \pi n y \delta_{\nu}\right] \\
& \cdot \exp \left[j 2 \pi\left(x+x_{0}\right)\left(m+p_{m n}\right) \delta_{\mu}\right] \cdots
\end{aligned}
$$

In the reconstructed image plane, we can get the original object wave $h(x, y)$. If the illumination light source is on-axis (i.e., $x_{0}=0$ ). Eq. $(7)$ can be more simplified as

$$
\begin{aligned}
& h(x, y)=\iint_{-\infty}^{+\infty} H(\mu, \nu) \exp [j 2 \pi(\mu x+\nu y)] d \mu d \nu \\
& =\sum_{m} \sum_{n} l_{m n} \| \dot{\delta}_{\mu} \dot{\delta}_{\nu} \operatorname{sinc}\left[l_{m n} y \delta_{\nu}\right] \\
& \sin c\left[I r \delta_{\mu}\right] \exp \left[j 2 \pi n y \delta_{\nu}\right] \\
& \exp \left[j 2 \pi x\left(m+p_{m n}\right) \delta_{\mu}\right] \\
& h(x, y)=\sum_{m} \sum_{n} l_{m n} \Pi \delta_{\mu} \delta_{\nu} \frac{\sin \left[\pi l_{m n} y \delta_{\nu}\right]}{\pi l_{m n} y \delta_{\nu}} \\
& \frac{\sin \left[\pi \Pi^{+} x \delta_{\mu}\right]}{\pi \Pi^{+} x \delta_{\mu}} \\
& \cdot \exp \left\{j 2 \pi\left[\left(m+p_{m n}\right) \cdot x \delta_{\mu}+n y \delta_{\nu}\right]\right\}
\end{aligned}
$$

According to $\mathrm{Eq} .(9)$, if the parameters of $p_{m n}, l_{m n}$. IF and the illumination source $s\left(x_{0}, y_{0}\right)$ can be properly chosen, the object wave $f(x, y)=A(x, y) \exp [j \varphi(x, y)]$ of $h(x, y)$ can be reconstructed by a computer.
Therefore, in terms of the above derivation, the complete numerical reconstruction is calculated procedure: (1) sampling and digitization of a $\mathrm{CGH} h(x, y),(2)$ calculation of the digital Fourier transform $H(\mu, \nu)$ from digitized hologram, (3) calculation of the product $H(m, n)$ of Fourier transforming integral. (4) calculation of the inverse digital Fourier transform of $H$ corresponding to the image $O(x, y)$ of the original object as shown in Fig, $6(6) \sim(9)$.

\section{Experiment Analyses and Discussion}

\subsection{Compression Efficiency and Distortion} Measure There are subjective criteria to decide if an image is distorted or not: the observation by the eye, and the comparison between the original image and the processed image. But it is not enough to determine objectively the quality of the image. That is why we must bring up the concept of MSE and PSYR.

According to the image processing theories, Eqs.(10), (11) and (12) of the compression ratio and the distortion measure can be written (10). R, MSE and PS.YR of the processed CGH in this system can be precisely calculated. Their relationship curves can be drawn as shown in Fig. 15 and 16.

$$
R=\frac{S_{c}}{S_{0}}
$$

Where $R$ is the compression ratio, $S_{0}$ is defined as the size of the original image data and $S_{c}$ is the size of the compressed image data.

$$
\begin{aligned}
& M S E=\frac{1}{512^{2}} \sum_{x=1}^{x=512} \sum_{y=1}^{y=512}[f(x, y)-\bar{f}(x, y)]^{2} \\
& P S Y R=10 \log _{10}\left[\frac{x_{p}^{2}}{M S E}\right](d B) \ldots \ldots \ldots . .
\end{aligned}
$$

Where $x, y$ are $1 \sim 512 . f(x, y)$ is CGH's image function (or reconstructed image function of original $\mathrm{CGH}$ ), $\bar{f}(x, y)$ is the decompressed CGH's image function (or reconstructed image function of decompressed $\mathrm{CGH}$ ). $x_{p}$ is 255 (peak to peak value of the image data).

5.2 CGH Compressed, Decompressed and Compression Ratio According to Eq. (10), the experiment data of compressed, decompressed and compression ratio $(R \%)$ of CGH can be calculated as shown in Table 2. The original CGH, compressed CGH, decompressed $\mathrm{CGH}$ and reconstructed image of $\mathrm{CGH}$ have been shown in Fig. $\sim \sim 11$. The histograms of original and decompressed CGH have been shown in Fig. $12 \sim 14$.

To analyze Fig. $i \sim 14$, we can find that the information distribution shape of 9 points diffraction fringe isn't destroyed as comparing Fig. 7 with Fig.9. And that the information distribution positions of both the reconstructed original image of CGH and the reconstructed image of processed $\mathrm{CGH}$ aren t raried as comparing Fig.10 with Fig.11. Moreorer, as comparing Fig.12 with Fig. 13, 14, we have found that the information field intensity distribution of $\mathrm{CGH}$ is reduced the dilution. and there is a little noise. But the information distribution 


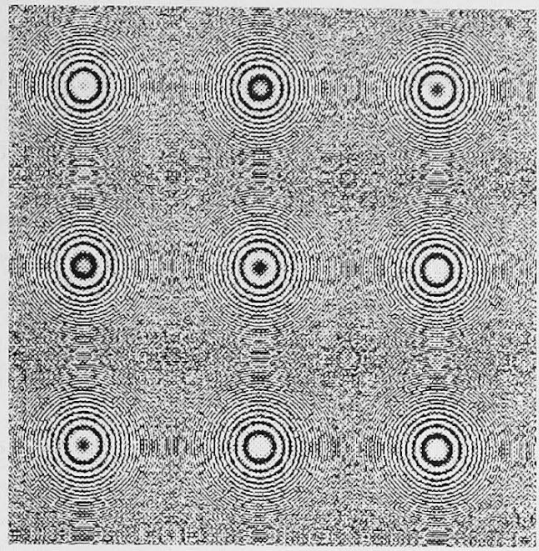

Fig. 7. Original CGH $\left(\right.$ size:7.11 $\times$ 7.11 inch $^{2}$ $512 \times 512$ pixel $\left.^{2}, 25 i \mathrm{~kb}, 8 \mathrm{bpp} / 256\right)$.

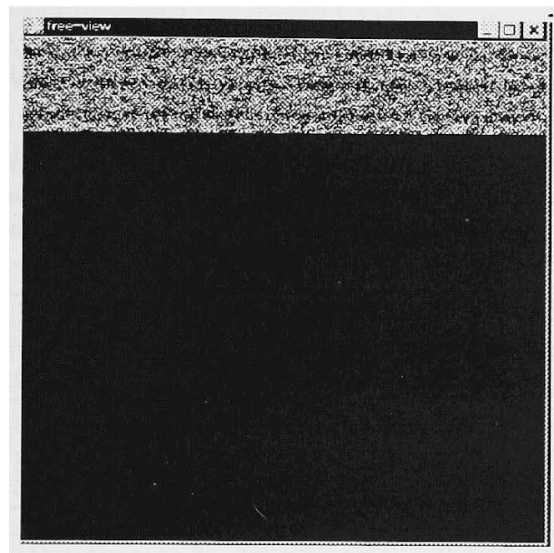

Fig. 8. Compressed CGH (size.7.11 $\times 7.11$ inch $^{2}$ $512 \times 512$ pixel $^{2} . \quad 48 \mathrm{~kb} . \quad 8 \mathrm{bpp} / 256 . \quad \mathrm{e}(\mathrm{N})=90$. $R=18.78 \%$

shape and position isn t varied and the image of the processed CGH can be reconstructed in limited value range. Therefore, we can evaluate that the reconstructed information distribution of the original and processed CGH is basically identical. In Fig.12, 13, 14, the histograms can be clearly seen about the frequency variation of processed CGH. In Sect.1 and Sect.4, the analyzed theories have effectively been verified by experiment images.

Table 2. Experiment data of compressed, decompressed and compressed ratio of $\mathrm{CGH}$

\begin{tabular}{|c|c|c|c|}
\hline$\varepsilon(N)=10$ & $C G H_{\text {original }}$ & $C G H_{c o m p r e s s e d}$ & $C G H_{d e c o m p r e s s e d}$ \\
\hline Size & $257 \mathrm{~kb}$ & $117 k b$ & $257 k b$ \\
\hline$R(\%)$ & & 45.4904 & 100 \\
\hline$E(N)=30$ & $C G H_{\text {ariginal }}$ & $C G H_{\text {compressed }}$ & $C G H_{d e c o m p r e s s e d}$ \\
\hline Size & $257 \mathrm{~kb}$ & $78 k b$ & $257 \mathrm{~kb}$ \\
\hline$R \mid \%$ & & 30.212 & 100 \\
\hline$\varepsilon(N)=60$ & CGHoriginal & CGH $H_{\text {compressed }}$ & $C G H_{d \varepsilon c o m p r e s s \in d}$ \\
\hline Size & $257 k b$ & $58 k b$ & $257 \mathrm{~kb}$ \\
\hline$R(\%)$ & & 22.369 & 100 \\
\hline $5(N)=70$ & $C G H_{\text {original }}$ & CGH compressed & $C G H_{d e c o m p r e s s e d}$ \\
\hline Size & $257 \mathrm{~kb}$ & $53 k b$ & $257 k b$ \\
\hline$R(\%)$ & & 20.4162 & 100 \\
\hline$\epsilon(X)=90$ & CGHoriginal & CGH compressed & $C G H_{d e c o m p r e s s e d}$ \\
\hline Size & $257 k b$ & $48 k b$ & $25 \div \mathrm{kb}$ \\
\hline$R \%$ & & 18.7806 & 100 \\
\hline
\end{tabular}

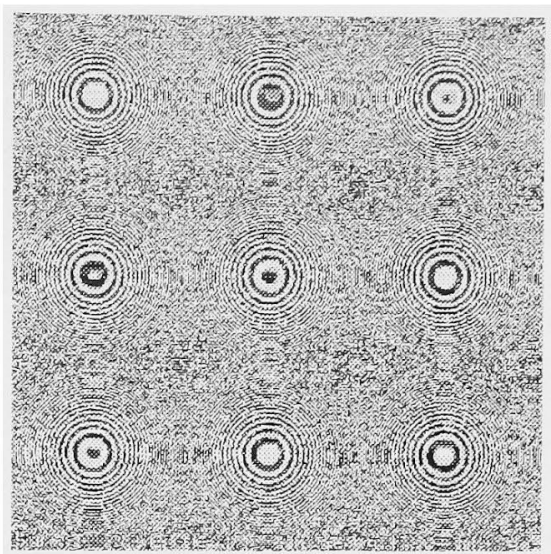

Fig. 9. Decompressed CGH (size:7.11 × 7.11 inch $^{2}$ $512 \times 512$ pixel $^{2}, \quad 257 \mathrm{~kb}, \quad 8 \mathrm{bpp} / 256 . \quad \mathrm{e}(\mathrm{N})=90$. $R=100 \%$.

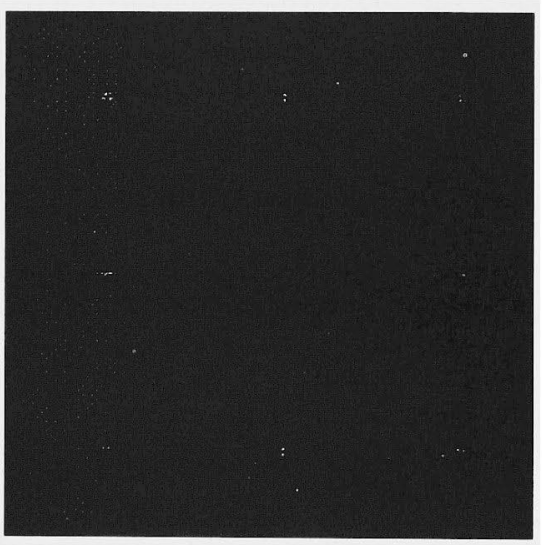

Fig. 10. Reconstructed image of original CGH using Fraunhofer transforming technique (size: $7.11 \times 7.11$ inch $^{2}, 512 \times 512$ pixel $\left.^{2}, 257 \mathrm{~kb}, 8 \mathrm{bpp} / 256\right)$

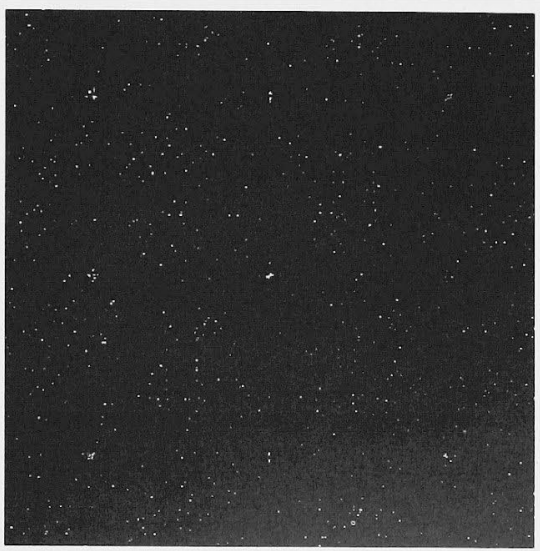

Fig. 11. Reconstructed image of decompressed $\mathrm{CGH}$ using Fraunhofer transforming technique (size: $7.11 \times 7.11$ inch $^{2}, 512 \times 512$ pixel ${ }^{2}, 25 \mathrm{tkb}$. $\mathrm{e}(\mathrm{N})=90) .8 \mathrm{bpp} / 256$.

5.3 CGH Transmitted Error and Reconstructed Image Quality In Table 3, the relation- 


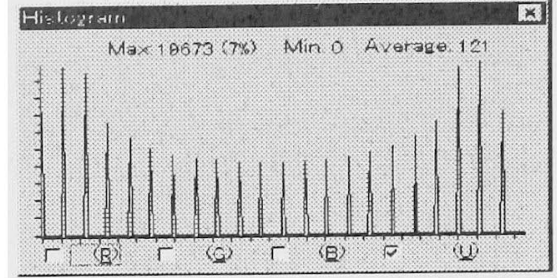

Fig. 12. Histogram of original CGH.

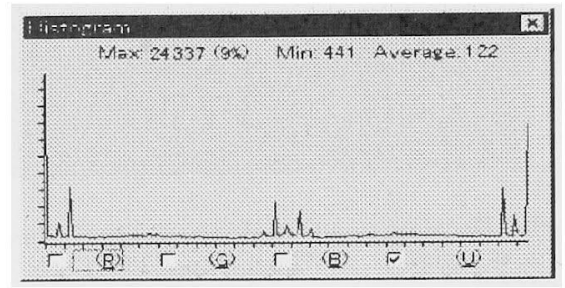

Fig. 13. Histogram of decompressed CGH $(e(N)=60)$.

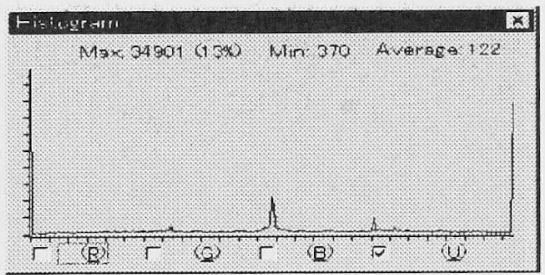

Fig. 14. Histogram of decompressed CGH $(e(N)=90)$.

Table 3. Relationship data about R\%. MSE and $P S{ }^{\circ} R$ under comparing the original CGH with the decompressed CGH.

\begin{tabular}{l|c|c|c|c|c}
\hline$E I N)$ & 10 & 20 & 30 & 40 & 50 \\
\hline$R(\%)$ & 45.4904 & 35.566 & 30.212 & 25.815 & 23.872 \\
\hline$I S E$ & 36.26925 & 133.124 & 283.567 & 481.522 & 728.564 \\
\hline$P S N R$ & 32.5354 & 26.888 & 23.604 & 21.305 & 19.506 \\
\hline$E I N)$ & 50 & 70 & 80 & 90 & 95 \\
\hline$R I \%)$ & 22.369 & 20.4162 & 19.607 & 18.7806 & 18.159 \\
\hline$I S E$ & 1021.692 & 1365.6864 & 1686.982 & 2068.1426 & 2394.260 \\
\hline$P S N R$ & 18.038 & 16.7773 & 15.859 & 14.9749 & 14.339 \\
\hline
\end{tabular}

ship data about $R(\%), M S E$ and PS.TR under comparing the original CGH with the decompressed CGH are described. $\epsilon(X)$ is defined as the parameter range for lossless coding $N=0$, range $=$ maxral +1 , for near-lossless coding $Y>0$, rang $=\left\lfloor\frac{\max \iota^{\prime} a l+2 * N}{2 * \Lambda+1}\right\rfloor+1$ in LOCO-I (5) † The relationship curres are shown in Fig. 15.

To analyze Fig. 15, the more precision relationship of this system can be found from $R, M S E$ and $P S Y R$ of CGH compressed and reconstructed. The "lossless" or "near-lossless" compression algorithm model can be criticized by these parameter data. In terms of Fig. 15 we can understand that IISE is directly proportional to $R(\%) . P S . R$ is inversely proportional to $R(\%)$

In Table 4, the relationship data about $R(\%)$. MSE and $P S Y R$ under comparing the reconstructed image of original CGH with the reconstructed image of decompressed CGH are described. The relationship curres are

\footnotetext{
†eg.. nlocoe-eiN, CGH.pgm: Compresses CGH.pgm in LI mode
} with loss $=N$, i.e. maximal allowed error $+/-N$.

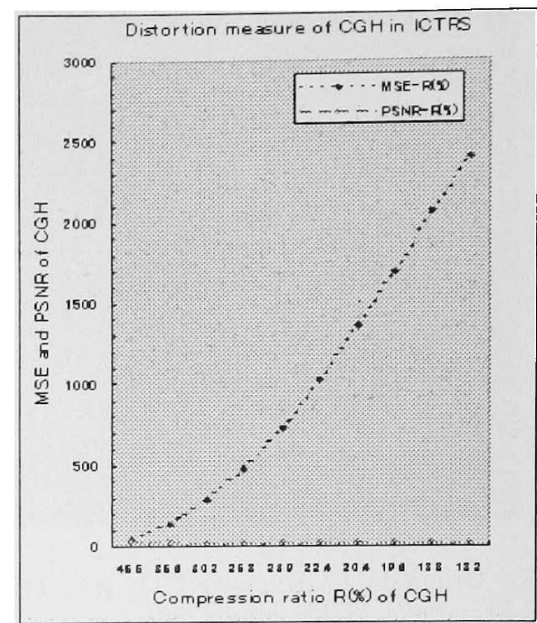

Fig. 15. Relationship curves about MSE, PSNR and $R(\%)$ in Comparing the original CGH with the decompressed CGH in ICTRS.

Table 4. Relationship data about R(\%), MSE and $P S Y R$ under comparing the reconstructed images of original CGH with the reconstructed images of decompressed CGH.

\begin{tabular}{c|c|c|c|c|c}
\hline$\varepsilon(N)$ & 10 & 20 & 30 & 40 & 50 \\
\hline$R(\%)$ & 45.4904 & 35.566 & 30.212 & 25.815 & 23.872 \\
\hline$I S E$ & 0.3867 & 1.07343 & 2.13498 & 3.4446 & 5.0774 \\
\hline$P S N R$ & 52.2571 & 47.8231 & 44.8369 & 42.7594 & 41.0744 \\
\hline$\varepsilon(N)$ & 60 & 70 & 80 & 90 & 95 \\
\hline$R(\%)$ & 22.369 & 20.4162 & 19.607 & 18.7806 & 18.159 \\
\hline$M S E$ & 7.5525 & 9.29696 & 10.8894 & 12.35302 & 13.9103 \\
\hline$P S N R$ & 39.3499 & 38.4474 & 37.7608 & 37.21307 & 36.6974 \\
\hline
\end{tabular}

shown in Fig.16. The quality of reconstructed images of $\mathrm{CGH}$ is inversely proportional to $R(\%)$.

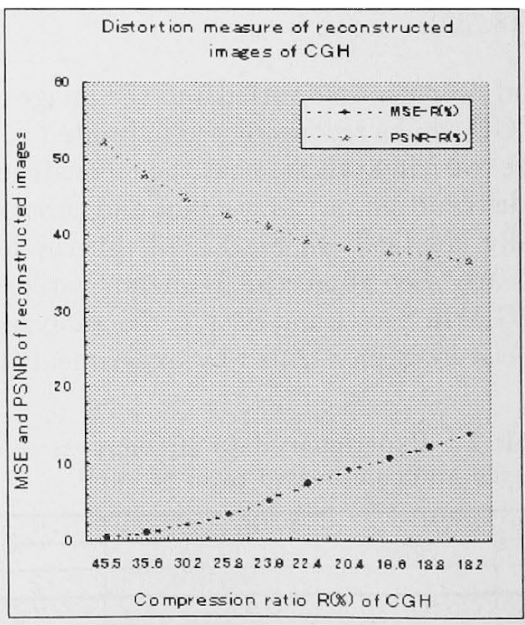

Fig. 16. Relationship curres of R(\%). MSE and $P S N^{\circ} R$ in comparing the reconstructed images of original CGH with the reconstructed images of decompressed $\mathrm{CGH}$

\section{Conclusion}

In this paper, the information compressed and transmitted and reconstructed software sistem (ICTRS) of 
CGH has been designed and established. This system can reconstruct and display the bigger images (i.e., $7.11 \times 7.11$ inch $^{2}$ ), whose compression ratio can be achieved to 15 . and the original image of CGH can be effectively reconstructed by ICTRS. The experiment results have effectively verified the theory discussed in Sect.1, that is the amplitude information of $\mathrm{CGH}$ has been reduced and the information of compressed phase can be effectively restored. To compare the reconstructed image (i.e., Fig.10) of original CGH with the one (i.e., Fig. 11) of processed CGH, the processed CGH can be effectively reconstructed by ICTRS

In processing $\mathrm{CGH}$, if the lost information of $\mathrm{CGH}$ exceeds certain limited value, its original information distribution will be raried (i.e., the limit values of compressed CGH must be controlled in $0 \leq \epsilon(N) \leq 90$ and $18.7806 \% \leq R(\%) \leq 100 \%$ in Table 3, 4.). And the reconstructed image of processed CGH can be directly observed by human risual system. The intensity $I(x, y)$ of the elements distribution of $\mathrm{CGH}$ has been decreased by the amplitude information compressed. But the original information distribution of $\mathrm{CGH}$ isn $\mathrm{t}$ distorted. The experiment results have verified that LOCO-I image encoding algorithm can satisfy the conditions of CGH reconstructed (i.e., the variation limit values of the amplitude and phase of CGH pixels compressed).

Potential applications of this system include the instrument complex using facsimile equipment for digital hologram input and recording (14), and the radar-guided system. In the future, perhaps it may be applied in the holography movie system ${ }^{(15)}$ and the coding techniques for holographic data storage systems (15).

In our experiments, we have chosen the symmetrical and the asymmetrical image of CGH to do the experiments. However in order to explain simply the image quality of $\mathrm{CGH}$ compressed and transmitted and reconstructed, we have analyzed the simmetrical one. Anyway, the experiment results have told us: we can use only computer software to complete effectively the total process of $\mathrm{CGH}$ compressed and transmitted and reconstructed

(Manuscript received February 23, 12, revised May 25. 12)

\section{References}

(1) Guanglin Yang and Eiji Shimizu. "Information, Compression and Transmission and Reconstruction of CGH with JPEG Image Processing." Technical Report of IEICE. OFSGG-37. IE99-46. pp.9-16. Sept. 17, 1999.

12, M. Weinberger. G. Seroussi, G. Sapiro. "LOCO-I: A Low Complexity: Context-Based, Lossless Image Compression Algorithm. Proc. IEEE Data Compression Conference. Snowbird. Utah. March-April 1995

131 M Weinberger G Seroussi G. Sapiro "The LoCO-I Lossless Image Compression Algorithm: Principles and Standardization into JPEG-LS." Hewlett-Packard Laburatories Techniral Report No. HPL-18-193. November 1998.

4. Marcelo J. Weinberger and Gadiel Seroussi. "From LOCO I to the JPEG-LS Standard." Computer Systems Laboratory Technical Reports. HP Laboratories Palo Alto. January 19 gG

15 FCD 14495. Lossless and near-lossless coding of continuous tone still images iJPEG-LSi." ISO/IEC JTC1/SC29 WWG1 (JPEG/JBIG), July 16, 199-'.

(6) Zuliang lu and Guofan Jin. "Computer Generated Hologram." Tsinghua University Publication Pressed, pp.33-35. (1984).

(7) WaiHon Lee. "Computer-Generated Holograms: Techniques and Applications." E. Wolf. Progress in Optics XVI @ NorthHolland 1978 .

(8) A.W Lohmann and D.P.Paris. "Binary. Fraunhofer Hologram Generated by Computer." Applied Optics. Vol.6. No.10. pP. 1739, October 195 ?

(9) Joseph W. Goodman. "Introduction to Fourier Optics," Department of Electrical Engineering. Stanford University

(10) WeiDong Kou. "Digital Image Compression Algorithms and Standards." Copyright (c) 1995 by Kluwer Academic Publishers. Boston/Dordrecht/London, pp.149-150.

111. Kenko Sasaki, Eichiro Tanji. Hiroshi Yoshikawa, "Data Compression for Holographic 3D Image." The journal of the Institute of Television Engineers of Japan. 48,10, pp. 1238-1244 Oct. 1994

112, Hiroshi loshikawa. Eichiro Tanji: "Method of Holographic 3-D Image Compression with a Standard Coding." The journal of the Institute of Television Engineers of Japan. 47,12 pp.1678-1680. Dec. 1993

(13) Lucente Mark, "Holographic bandwidth compression using spatial subsampling," Optical Engineering, vol.35, No.5. pp 1599-1537. June 1996.

(14) M.P.Grishin. Sh.MKurbanov, and Y.P.Markelov. "Automatic computer entry and processing of photographic images." in Russian, Énergiya. Moscow, 1975 .

(15) Vgl. Victor Komar, "Progress on the Holographic Movie Pro cess." in the USSR, in SPIE. Bd. 120, S. 12T-144, 197.

(16) Jonathan J. Ashley. Mario Blaum and Brian H. Marcus, "Report on Coding Techniques for Holographic Storage." IBMI Research Report, Published in RJ10013. 1996.

Guanglin Yang (Non-member) is studying for

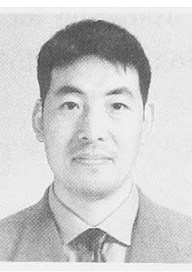
Ph. D. degree in Faculty of Electrical Engineering: Osaka City University; in Japan. He received M. E. degree from Huazhong University of Science \& Technology, China, in 1993. He was a lecturer in Faculty of Automatic Control Engineering, Beijing University of Aeronautics and Astronautics. China. in 1994. His research interests mainly include the optical information processing. computer generated hologram. image processing, intelligent infrared detecting system, computer aid design $(C .+D)$ automatic control system and digital control technique

Eiii Shimizu (Nember) receired B. S. degree

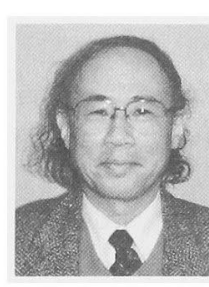
from Department of Electrical Engineering in Osaka University; Japan, in 1963. He receired Dr. Eng. degree from Osaka University: From 1963. he was an assistant in Department of Electrical Engineering. Osaka City University, and he became a professor in 1983 . His research interests mainly include the electronic circuit, the optical electron techniques, and the optical information processing and neural networks. He respectively is a member of IEEE, SPIE, and the Institute of Electrical Engineers of Japan, the Institute Telerision Engineers of Japan and the Institute of Sistems, Control and Information Engineers of Japan 